\title{
Approaches to identifying drug resistance mechanisms to clinically relevant treatments in childhood rhabdomyosarcoma
}

\author{
Samson Ghilu ${ }^{1}$, Christopher L. Morton ${ }^{2}$, Angelina V. Vaseva ${ }^{1}$, Siyuan Zheng ${ }^{3}$, Raushan T. Kurmasheva ${ }^{1}$, \\ Peter J. Houghton ${ }^{1}$ \\ 'Department of Molecular Medicine, Greehey Children's Cancer Research Institute, UT Health, San Antonio, TX 78229, USA. \\ ${ }^{2}$ Department of Surgery, St. Jude Children's Research Hospital, Memphis, TN 38105, USA. \\ ${ }^{3}$ Department of Epidemiology and Biostatistics, Greehey Children's Cancer Research Institute, UT Health, San Antonio, TX \\ 78229 , USA
}

Correspondence to: Dr. Peter J. Houghton, Department of Molecular Medicine, Greehey Children's Cancer Research Institute, UT Health, San Antonio, 8403 Floyd Curl Drive, San Antonio, TX 78229, USA. E-mail: HoughtonP@uthscsa.edu

How to cite this article: Ghilu S, Morton CL, Vaseva AV, Zheng S, Kurmasheva RT, Houghton PJ. Approaches to identifying drug resistance mechanisms to clinically relevant treatments in childhood rhabdomyosarcoma. Cancer Drug Resist 2022;5:80-9. https://dx.doi.org/10.20517/cdr.2021.112

Received: 11 Oct 2021 First Decision: 23 Nov 2021 Revised: 3 Dec 2021 Accepted: 15 Dec 2021 Published: 4 Jan 2022

Academic Editors: Godefridus J. (Frits) Peters, Brian A. Van Tine Copy Editor: Yue-Yue Zhang Production Editor: Yue-Yue Zhang

\begin{abstract}
Aim: Despite aggressive multiagent protocols, patients with metastatic rhabdomyosarcoma (RMS) have poor prognosis. In a recent high-risk trial (ARST0431), 25\% of patients failed within the first year, while on therapy and $80 \%$ had tumor progression within 24 months. However, the mechanisms for tumor resistance are essentially unknown. Here we explore the use of preclinical models to develop resistance to complex chemotherapy regimens used in ARST0431.
\end{abstract}

Methods: A Single Mouse Testing (SMT) protocol was used to evaluate the sensitivity of 34 RMS xenograft models to one cycle of vincristine, actinomycin D, cyclophosphamide (VAC) treatment. Tumor response was determined by caliper measurement, and tumor regression and event-free survival (EFS) were used as endpoints for evaluation. Treated tumors at regrowth were transplanted into recipient mice, and the treatment was repeated until tumors progressed during the treatment period (i.e., became resistant). At transplant, tumor tissue was stored for biochemical and omics analysis. 
Results: The sensitivity to VAC of 34 RMS models was determined. EFS varied from 3 weeks to $>20$ weeks. Tumor models were classified as having intrinsic resistance, intermediate sensitivity, or high sensitivity to VAC therapy. Resistance to VAC was developed in multiple models after 2-5 cycles of therapy; however, there were examples where sensitivity remained unchanged after 3 cycles of treatment.

Conclusion: The SMT approach allows for in vivo assessment of drug sensitivity and development of drug resistance in a large number of RMS models. As such, it provides a platform for assessing in vivo drug resistance mechanisms at a "population" level, simulating conditions in vivo that lead to clinical resistance. These VACresistant models represent "high-risk" tumors that mimic a preclinical phase 2 population and will be valuable for identifying novel agents active against VAC-resistant disease.

Keywords: Rhabdomyosarcoma, patient-derived xenografts, combination therapy, intrinsic drug resistance, acquired drug resistance

\section{INTRODUCTION}

Advanced or metastatic rhabdomyosarcoma (RMS) has a poor prognosis. Patients with stage 4 disease, with the exception of those with embryonal RMS that are less than ten years of age, have a long-term event-free survival (EFS) of less than $20 \%^{[1-3]}$. Current therapies include all the known active agents plus radiation and surgery, but neither intensification of chemotherapy by increasing the dose of cyclophosphamide and adding active agents to standard VAC therapy (Vincristine/Actinomycin-D/Cyclophosphamide), nor use of high-dose chemotherapy with stem-cell rescue has improved outcome over the last 30 years ${ }^{[4]}$. The failure of current therapeutic approaches to effectively treat advanced or metastatic RMS despite numerous cooperative group trials, is a consequence of intrinsic or acquired resistance to the limited number of drugs that compose the armamentarium for treating this disease. Even with the use of intensive multimodality treatment, only $20 \%$ of patients will be long-term survivors. The failure of current therapies is further emphasized by the rapid progression rate of patients enrolled in a recent high-risk rhabdomyosarcoma study (ARST0431). In this trial, 25\% of patients diagnosed with metastatic disease, either failed during the 52 weeks of treatment or had an event within two years $(80 \%)^{[5]}$. The causes of tumor resistance are poorly understood. One possible cause for resistance is tumor clonal evolution, where novel mutations acquired during therapy confer resistance ${ }^{[6]}$; however, such studies usually rely on small cohorts as relatively few tumors are re-biopsied at relapse.

Preclinical studies aimed at understanding resistance tend to focus on individual drugs, rather than resistance to poly-chemotherapy ${ }^{[7-13]}$. Further, often drug exposures used in vitro far exceed those achieved in patients, and escalating drug concentrations over time do not simulate how clinical resistance is acquired $^{[14]}$. We have previously used pediatric tumor xenografts to select for acquired resistance in mice ${ }^{[7,8]}$. However, while this approach is perhaps more appropriate than in vitro selection, conventional testing is resource-intensive, and allows for only a few models to be explored ${ }^{[15]}$. More recently, we have adopted Single Mouse Testing (SMT) to evaluate drugs in a large number of xenograft models ${ }^{[16,17]}$, an approach that significantly increases the inclusion of genetic diversity for a given cancer type ${ }^{[18]}$. The SMT design is validated by a retrospective analysis of $>2100$ tumor-drug studies undertaken by the Pediatric Preclinical Testing Program, where the response of a tumor in one mouse, selected at random from the group, was compared to the median group response. This analysis showed that the SMT accurately predicted responses in $78 \%$ of studies. Allowing for a deviation of \pm one response classification [e.g., stable disease (SD) vs. partial response $(\mathrm{PR})$ ], the concordance was 95\%. Further, the SMT analysis was accurate in identifying the antitumor activity of 66 of 67 drugs in terms of the objective response rate determined for each drug over a range of tumor models ${ }^{[18]}$. Prospective studies with up to 90 ALL models and up to 50 solid tumor models 
show that SMT has similar concordance with conventional testing ${ }^{[16]}$. Importantly, using SMT, one can potentially incorporate up to 20 -fold the number of models for evaluation of an agent, encompassing many diseases, or encompassing the genetic diversity of a given disease. Here we have applied SMT to interrogate intrinsic resistance and to use this approach to select for acquired resistance in vivo to VAC therapy in 34 RMS models. Our data show that RMS models have a range of sensitivities to VAC, ranging from tumor progression through initial treatment to maintained complete response at week 20 after a single cycle of VAC treatment. Further, using the SMT design, acquired resistance can be developed over a period of 3 to 5 cycles of therapy.

\section{METHODS}

C.B.17SC scid ${ }^{-1}$ (C.B-Igh-1 ${ }^{\mathrm{b}} / \mathrm{IcrTac}-P r k d c^{\text {scid }}$ ) female mice (Envigo, Indianapolis, IN) were maintained under barrier conditions, and experiments were conducted using protocols and conditions approved by the Institutional Animal Care and Use Committee at UTHSA as previously described ${ }^{[15]}$. Mice were selected for VAC treatment when tumors were $200-300 \mathrm{~mm}^{3}$. Regrowth of tumors was determined following tumor regression. Endpoints were EFS, defined as tumor growing to $400 \%$ of its volume at the initiation of treatment, and percent tumor volume regression. Complete regression (CR) was defined as tumor volume < $40 \mathrm{~mm}^{3}$ (the level of detection). Limited demographic data for RMS models used in the studies are presented in Table 1. Patient- and cell-derived xenograft models (PDX and CDX) have been described previously ${ }^{[1,17]}$. Genomic data for some of the models is under https://ocg.cancer.gov/programs/target/ Pancancer Model Systems, and in Rokita et al. ${ }^{[19]}$.SJRHB011_X, SJRHB013_X, SJRHB010927_X1, SJRHB000026_X1, and SJRHB013758_X1 were obtained from The Childhood Solid Tumor Network ${ }^{[20]}$. SMS-CTR cells were obtained from Dr. Corine Linardic, Duke University, JR1 (UK), and CCA cells were obtained from Dr.Marielle Yohe, NCI. All tumors were authenticated by short tandem repeat analysis against reference profiles.

\section{Simulating VAC therapy in mice}

All drugs were administered IP. Vincristine was administered at $0.5 \mathrm{mg} / \mathrm{kg}$ days $1,8,15$, actinomycin D $(0.20 \mathrm{mg} / \mathrm{kg})$ and cyclophosphamide $(120 \mathrm{mg} / \mathrm{kg})$, both on day 1 , doses considered to give clinically relevant drug exposures ${ }^{[2-23]}$. All drugs were formulated in $0.9 \% \mathrm{w} / \mathrm{v}$ saline. This regimen, which simulates VAC modules used in ARST0431, was tolerated with $\sim 10 \%$ body weight loss, and mortality $<2 \%$.

\section{Development of acquired resistance in mice}

The schema for selecting for acquired resistance is presented in Figure 1. Briefly, one mouse per tumor line received a single cycle of VAC treatment. Tumor diameters were measured weekly, and treated tumors were re-transplanted into new recipient mice when they "evented" (achieved $400 \%$ of their volume on day 1 of treatment). Treated tumors were considered resistant to VAC therapy if they progressed through treatment and had increased $>25 \%$ in volume by day 21 . Tumor tissue was cryopreserved and snap frozen after each cycle of VAC therapy.

\section{RESULTS}

\section{Intrinsic sensitivity to VAC treatment}

We initially explored the sensitivity of 34 RMS xenograft models to the "gold standard" therapy for RMS, namely VAC, Figure 2. The models encompass both fusion-positive (alveolar), and fusion-negative (embryonal, with and without RAS mutations). As shown in Figure 2A, 18 of 34 xenograft models (53\%) regressed $>50 \%$ on Cycle 1 of VAC therapy, the other models showing SD $(n=3)$ or progressive disease (PD) $(n=13)$. This experiment is ongoing, but clearly shows that SMT can be valuable for assessing drug sensitivity in a large cohort of models. Thirteen models were intrinsically resistant to VAC treatment with EFS of $<40$ days, whereas others $(n=10)$ are highly sensitive (EFS $>70$ days), with four models remaining 
Table 1. Characteristics of RMS xenograft models and response to cycle 1 of VAC therapy

\begin{tabular}{|c|c|c|c|c|c|c|c|}
\hline Model ID & Subclass & $\begin{array}{l}\text { Age } \\
\text { (years) }\end{array}$ & Gender & Site & $\begin{array}{l}\text { Diagnosis or } \\
\text { relapse }\end{array}$ & Response & $\begin{array}{l}\text { EFS } \\
\text { (weeks) }\end{array}$ \\
\hline Rh10 & ARMS & 15 & Female & Liver & Relapse & PD & 6 \\
\hline Rh28 & ARMS & 17 & Male & Hand & Diagnosis & $M C R$ & $>20$ \\
\hline $\mathrm{Rh} 30 \mathrm{R}$ & ARMS & 16 & Male & Bone marrow & Relapse & $C R$ & 9 \\
\hline Rh41 & ARMS & 12 & Female & Unknown & Autopsy & PD & 3 \\
\hline Rh18 & ERMS & 2 & Female & Perineum & Diagnosis & $\mathrm{CR}$ & 10 \\
\hline Rh36 & ERMS & 15 & Male & Paratesticular & Relapse & $P R$ & 6 \\
\hline Rh66 & ARMS & 12 & Female & Axillary lymph node & Metastasis & $\mathrm{CR}$ & 7 \\
\hline Rh12 & ERMS & 12 & Male & Right buttock & Diagnosis & $M C R$ & $>20$ \\
\hline NCH-ERMS-1 & ERMS & 5 & Male & orbital & No treatment & PD & 5 \\
\hline $\mathrm{NCH}-\mathrm{ARMS}-2$ & ARMS, metastatic & 16 & Female & Right breast & Treated & PD & 4 \\
\hline Rh71 & ERMS, high grade & 17 & Male & Prostate & Diagnosis & PD & 6 \\
\hline Rh72 & ERMS & 3 & Female & Perineal & Treated & PD & 9 \\
\hline Rh73 & ERMS & 5 & Male & $\begin{array}{l}\text { Right infratemporal fossa } \\
\text { mass }\end{array}$ & Treated & PD & 7 \\
\hline Rh75 & ERMS & 17 & Male & Pelvis recurrent (Rh71) & Treated & $\mathrm{CR}$ & 8 \\
\hline Rh78 & ARMS & 1 & Male & Right thigh & No treatment & SD & 8 \\
\hline Rh80 & ERMS anaplastic & 5 & Female & Stomach mass & Treated & PD & 5 \\
\hline Rh81 & ERMS & 9 & Male & Abdominal mass & Treated & PR & 7 \\
\hline Rh82 & ARMS & 3 & Male & Paratesticular & Treated & $P R$ & 10 \\
\hline Rh83 & ERMS & 4 & Male & Left orbital mass & Treated & PR & 14 \\
\hline Rh84 & ARMS & 2 & Male & Upper lip lesion & Treated & SD & 12 \\
\hline Rh85 & $\begin{array}{l}\text { ERMS diffuse } \\
\text { anaplasia }\end{array}$ & 5 & Female & Abdominal mass & No treatment & PD & 4 \\
\hline Rh86 & ERMS & 8 & Male & Retroperitoneal mass & Treated & $\mathrm{CR}$ & 10 \\
\hline Rh87 & $\begin{array}{l}\text { Spindle } \\
\text { cell/sclerosing }\end{array}$ & 6 & Female & Oropharyngeal mass & Treated & PD & 3 \\
\hline Rh88 & ERMS & 10 & Male & Pelvic mass & Treated & CR & 12 \\
\hline IRS-56 & ERMS & 3 & Male & Buttock & Diagnosis & $\mathrm{CR}$ & $>20$ \\
\hline IRS-68 & ERMS & 13 & Male & Shoulder & Diagnosis & PR & 7 \\
\hline SJRHB011_X & ERMS & 5 & Male & Infratemporal fossa & Recurrent & PD & 4 \\
\hline SJRHB013_X & ERMS & 3 & Female & Perineal/bladder & Recurrent & $\mathrm{CR}$ & 13 \\
\hline SJRHB010927_X1 & ERMS & 5 & Female & Parapharyngeal & Diagnosis & PR & 12 \\
\hline $\begin{array}{l}\text { SJRHB000026- } \\
\text { X1 }\end{array}$ & ERMS & 4 & Female & Pelvis & Recurrent & PD & 5 \\
\hline SJRHB013758_X1 & ERMS & 4 & Female & Abdomen/pelvis & Diagnosis & $C R$ & 10 \\
\hline SMS-CTR* & ERMS & 1 & Male & Pelvis & Diagnosis & MCR & $>20$ \\
\hline$J R-1(U K)^{\star}$ & ERMS & 7 & Female & Lung & Relapse & PD & 7 \\
\hline $\mathrm{RD}^{*}$ & ERMS & 7 & Female & Pelvis & Relapse & SD & 11 \\
\hline
\end{tabular}

*Indicates cell line derived xenograft. ARMS: Fusion-positive; ERMS: fusion negative; RMS: rhabdomyosarcoma; VAC: vincristine/actinomycinD/cyclophosphamide; EFS: event-free survival; PD: progressive disease; MCR: maintained complete response; CR: complete regression; PR: partial response; SD: stable disease.

in CR at 120 days, as shown in the Kaplan-Meier plot [Figure 2B]. We defined PD as tumors that increased in volume by $25 \%$ at day 21 ( 6 days after the last dose of vincristine). Thirteen of 34 RMS models (38\%) exhibited PD to cycle 1 of VAC. An EFS of 49 days or less was also observed for 13 models, Table 1. Of these 13 intrinsically resistant models, 10 tumors were established from patients following treatment. The exceptions were Rh71, Rh85, and NCH-ERMS1, which were established as PDX models from patients prior to any treatment. Other models with short EFS (CCA, Rh36, IRS-68) each regressed > 50\% (i.e., PR), but 


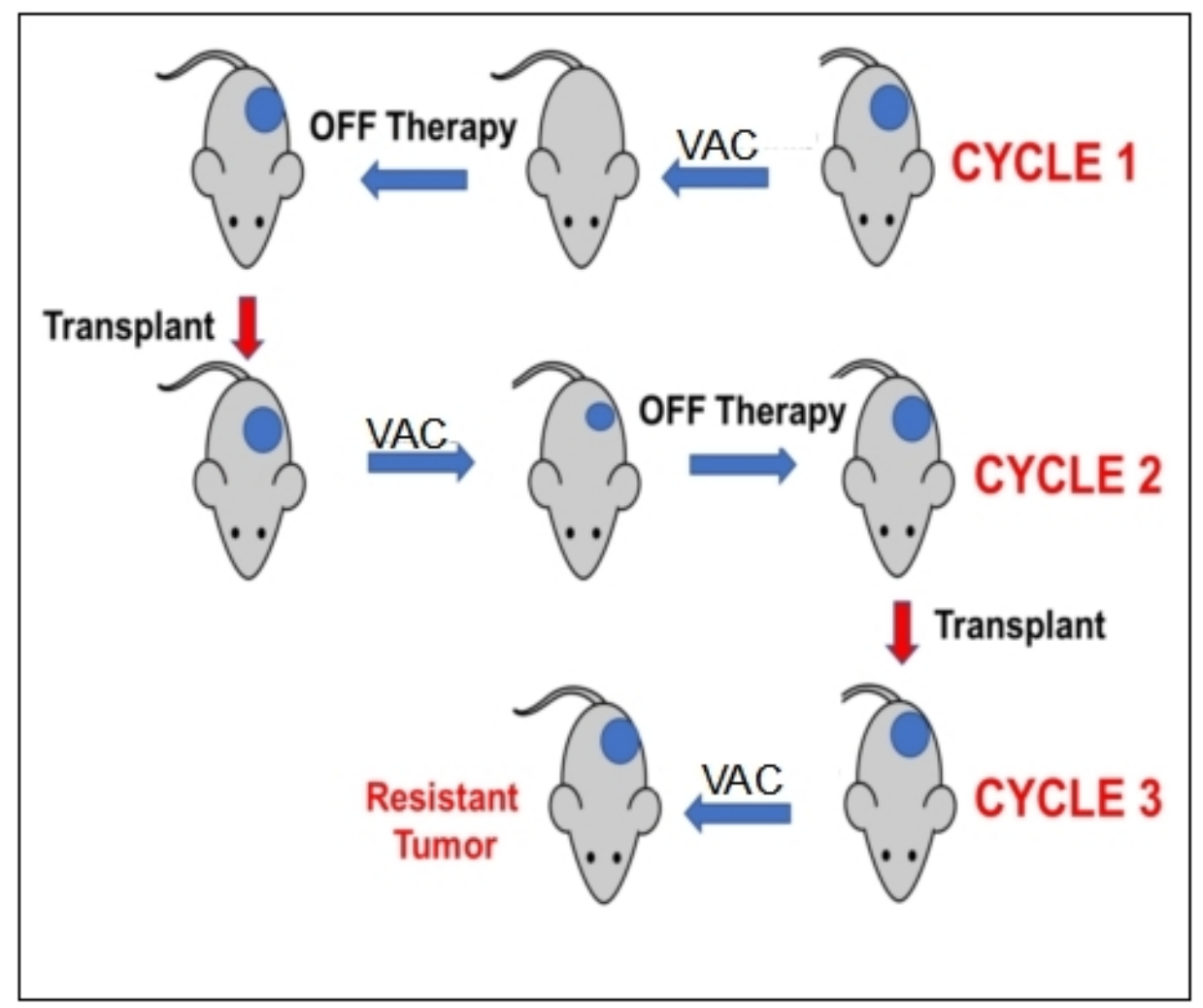

Figure 1. Schema for developing resistance to VAC therapy in mice using the single mouse study design. Mice received a single cycle of VAC (vincristine, actinomycin D, cyclophosphamide). Tumor response was determined, and tumors were transplanted in recipient mice when they achieved $400 \%$ of their volume on the first day of treatment.
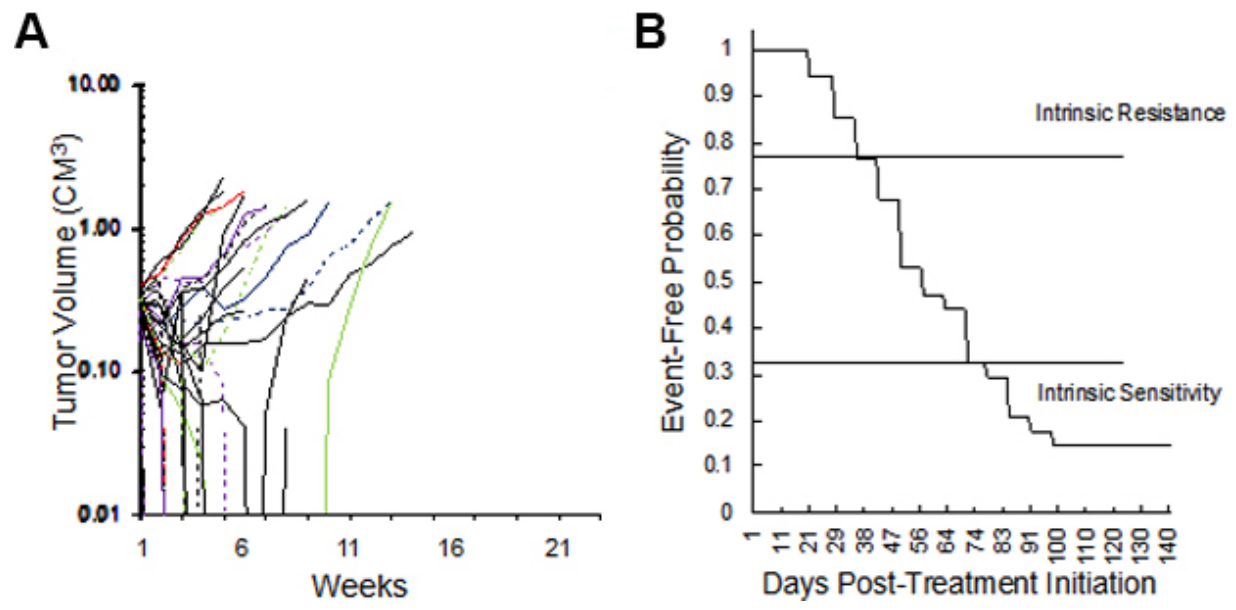

Figure 2. (A) Initial volume responses of 34 RMS models to cycle 1 of vincristine + actinomycin D + cyclophosphamide (VAC) using the SMT experimental design (ongoing expt.). (B) Kaplan-Meier plot for EFS of the same models. RMS: Rhabdomyosarcoma; VAC: vincristine/actinomycin-D/cyclophosphamide; EFS: event-free survival; SMT: single mouse testing.

rapidly regrew. Of the models having EFS of 70 days or greater $(n=10 ; 29 \%), 3$ models had CR (Rh88, SMSCTR, SJRHB013x, and SJRHB013758_X1), 2 had PR (SJRHB010927, Rh83), and 1 had prolonged SD (Rh84). Three models, established from tumor at diagnosis, were highly sensitive to VAC treatment with no evidence of tumor regrowth at day 120 after the start of treatment (IRS-56, Rh12, Rh28). The other 11 
models had intermediate sensitivity to VAC (EFS $>40$ but $<70$ days).

\section{Development of acquired resistance}

The approach to selecting for acquired resistance [Figure 1], was chosen as there is an increased incidence of scid lymphoma as mice age, and also potentially altered immunity that could alter the response to chemotherapy. Consequently, we re-transplanted tumors at $400 \%$ of their volume at day 1 of treatment into new 6 to 8 -week-old female C.B.17SC scid ${ }^{-1-}$ mice, and repeated this procedure until tumors became resistant. For all studies, tumors were allowed to grow to $200-300 \mathrm{~mm}^{3}$ before starting treatment. Initially, we transplanted cycle 1 tumor into two mice, in case there was toxicity, and we would lose the model if there was a death (drug related or unrelated) on cycle 2 of treatment. However, there was no mortality, so subsequently, a single mouse design was used for all subsequent cycles of VAC treatment. Where two mice were used $(n=16)$, the responses between tumors were very similar, Supplemental Figure 1. The "Swimmer plot" in Figure 3 shows examples of models exhibiting Complete Regression (Rh88) or Partial Regression (IRS-6) to VAC treatment on cycle 1 with rapid emergence of resistance, or tumor models that initially had progressive disease (i.e., intrinsically resistant), but where EFS shortened with further cycles of VAC treatment (Rh72), or a model where EFS remained constant during 3 cycles of VAC treatment (Rh73).

\section{DISCUSSION}

The outcome for patients with high-risk RMS has not improved significantly for several decades. In part, this is because new effective agents have not been identified, and agents such as irinotecan, while active against RMS ${ }^{[2,25]}$, do not increase overall survival when added to VAC therapy ${ }^{[5,26]}$. Of the chemotherapeutic agents used in high-risk protocols such as ARST0431, all but one (vincristine), induces DNA damage. However, the mechanism(s) that confer resistance to multi-chemotherapy protocols, even protocols that combine mostly DNA damaging agents, remains unknown. A proportion of patients failing on therapies including cyclophosphamide and vincristine ${ }^{[27]}$, however, may respond to vinorelbine/cyclophosphamide ${ }^{[28]}$.

Approaches to determine mechanisms of resistance, both in vitro and in vivo, have limitations. As mentioned previously, in vitro studies may not accurately replicate clinical drug exposures, and continuous exposure to increasing drug levels may result in multidrug resistance mechanisms, such as overexpression of $A B C$ transporters, that may not reflect clinical reality ${ }^{[29]}$. In vivo studies in mice, using human tumor xenografts, or syngeneic tumor models, may more accurately model drug pharmacokinetics relevant to clinical exposures. However, resource constraints tend to limit their use to a few models and usually for the development of resistance to single agents. One approach to encompassing genetic diversity for a given cancer type is to use an SMT design, rather than conventional experimental designs that use 8-10 mice for control and treatment group ${ }^{[1,1,18}$. In this pilot study, we have evaluated SMT in the context of VAC therapy in 34 RMS models, and have used this approach to develop models with acquired resistance that can be further characterized by "omics" and biochemical methods. Essentially, the approach mimics a clinical trial in that there is no "control" arm, and the response criteria are tumor volume changes and EFS. We "enrolled" RMS models when tumors were available, and the response to one cycle of VAC was assessed in a blinded manner with respect to whether the model was established from an untreated patient or from a patient following treatment. As VAC therapy has been the backbone for the treatment of RMS for over 30 years, we assume that xenografts established from treated patients received these drugs, although it is not known if additional drugs were administered to patients before their tumors were established as xenografts.

Overall the models appear to retain drug sensitivity characteristics of patient tumors. Ten of the 13 models that had PD to VAC treatment were derived from pre-treated tumors (treated, relapse, autopsy), whereas only 2 out of 11 models established from diagnosis samples progressed on cycle 1 of VAC treatment. The 


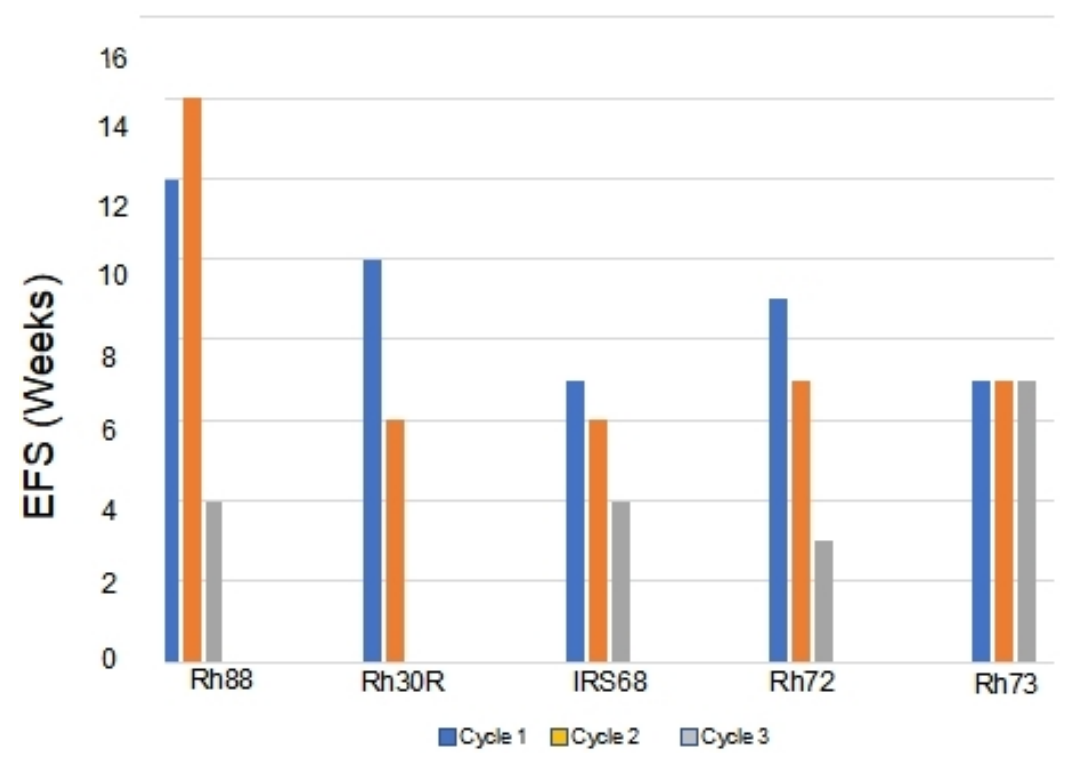

Figure 3. Development of resistance to VAC treatment (decreases in EFS) in models that were intrinsically sensitive to treatment (> PR; Rh88, Rh30R, IRS-68) or models where tumors progressed through cycle 1 of VAC treatment (Rh72, Rh73). VAC: Vincristine/actinomycin-D/cyclophosphamide; EFS: event-free survival; PR: partial response.

models established as PDX's from samples at diagnosis that were intrinsically resistant to VAC included two ERMS, one defined pathologically as a high-grade tumor (Rh71) and one with diffuse anaplasia (Rh85), which may relate to drug sensitivity ${ }^{[30,31]}$. Tumor models having EFS of 70 days or greater included 7 established from diagnosis samples, 5 listed as "treated", and 3 models established from tumor at relapse. Clinically, patients progressing on front-line treatment may respond to phase 2 protocols, including cyclophosphamide and vinca alkaloids. It is also possible that prolonged passage of tumor in untreated mice could lead to loss of resistance, or overgrowth of sensitive cells. However, the data set from 34 models serves as a reference point for understanding intrinsic resistance or sensitivity and for developing acquired resistance in those models that initially respond to VAC treatment under relevant in vivo conditions.

We have used two criteria for assessing tumor sensitivity to VAC: tumor regression (partial and complete response) and EFS. Tumors having PD had EFS times of 3-8 weeks; those demonstrating PR (> 50\% volume regression) had EFS that ranged from 6-14 weeks, and models having CR the EFS time ranged from 7 to $>$ 20 weeks. Thus, as in our previous study ${ }^{[17]}$, there was a relatively poor correlation between the magnitude of tumor volume regression and EFS, suggesting that EFS may be a more reliable metric of tumor response in preclinical models. Clinical data also question the value of early tumor response as a prognostic indicator, as neither computed tomography and/or magnetic resonance imaging predicted outcomes for patients with rhabdomyosarcoma ${ }^{[32,33]}$.

We have attempted to select for acquired resistance to VAC using the SMT approach. While this pilot study is far from complete, interim analysis suggests that this approach can generate a large number of VACresistant models that can be analyzed for genetic/epigenetic changes associated with resistance. Thirteen xenograft models showed progressive disease in response to VAC treatment, hence were classified as intrinsically resistant. However, many of these resistant tumors had reduced EFS on subsequent cycles of therapy, thus showing increased resistance. In ongoing studies, acquired resistance has been selected within 3 to 5 cycles of VAC treatment in many tumor models initially sensitive to VAC. The approach we have taken is to define intrinsic sensitivity/resistance to VAC and to select for acquired resistance in mice. An 
alternative approach is to establish PDX models at diagnosis and relapse from the same patient. This is rarely possible in the context of pediatric RMS, as relapse tumor is rarely biopsied, but also engraftment into mice may select for subclones rather than represent the clonal spectrum in the patient tumor. Modeling intrinsic and acquired resistance in preclinical models has an advantage over clinical studies in that changes in gene expression can be assessed both in parental and resistant tumor, but also in response to therapy ${ }^{[34]}$.

Here we have defined the intrinsic sensitivity of 34 RMS models. Importantly, models intrinsically resistant to VAC, or those with acquired resistance to VAC treatment, may be particularly valuable for identifying novel agents that may be active in disease at relapse, and may have novel mechanisms of action independent of DNA damage.

\section{DECLARATIONS}

\section{Acknowledgements}

We thank Dr. Abhik Bandyopadhyay for management and scheduling of these studies.

\section{Authors' contributions}

Made substantial contributions to conception and design of the study and performed data analysis and interpretation: Ghilu S, Morton CL, Vaseva AV, Zheng S, Kurmasheva RT, Houghton PJ

Performed data acquisition, as well as provided administrative, technical, and material support: Ghilu S, Morton CL, Houghton PJ

\section{Availability of data and materials}

All models are available to academic centers under an institutional MTA.

\section{Financial support and sponsorship}

Studies reported here were supported by CA23099, NO1-CM42216, UO1CA199297, RO1CA169368, PO1CA165995 and CPRIT RP160716.

\section{Conflicts of interest}

All authors declared that there are no conflicts of interest.

\section{Ethical approval and consent to participate}

Experiments were conducted using protocols and conditions approved by the Institutional Animal Care and Use Committee at UTHSA. Use of patient-derived tissue was considered non-human research by the IRB.

\section{Consent for publication}

Use of patient-derived tissue was considered non-human research by the IRB. All xenografts are deidentified.

\section{Copyright}

(c) The Author(s) 2022.

\section{REFERENCES}

1. Malempati S, Hawkins DS. Rhabdomyosarcoma: review of the Children's Oncology Group (COG) Soft-Tissue Sarcoma Committee experience and rationale for current COG studies. Pediatr Blood Cancer 2012;59:5-10. DOI PubMed PMC

2. Oberlin O, Rey A, Lyden E, et al. Prognostic factors in metastatic rhabdomyosarcomas: results of a pooled analysis from United States and European cooperative groups. J Clin Oncol 2008;26:2384-9. DOI PubMed PMC

3. Breneman JC, Lyden E, Pappo AS, et al. Prognostic factors and clinical outcomes in children and adolescents with metastatic rhabdomyosarcoma--a report from the Intergroup Rhabdomyosarcoma Study IV. J Clin Oncol 2003;21:78-84. DOI PubMed

4. Weigel BJ, Breitfeld PP, Hawkins D, Crist WM, Baker KS. Role of high-dose chemotherapy with hematopoietic stem cell rescue in the treatment of metastatic or recurrent rhabdomyosarcoma. J Pediatr Hematol Oncol 2001;23:272-6. DOI PubMed 
5. Weigel BJ, Lyden E, Anderson JR, et al. Intensive multiagent therapy, including dose-compressed cycles of ifosfamide/etoposide and vincristine/doxorubicin/cyclophosphamide, irinotecan, and radiation, in patients with high-risk rhabdomyosarcoma: a report from the Children's Oncology Group. J Clin Oncol 2016;34:117-22. DOI PubMed PMC

6. Chen X, Stewart E, Shelat AA, et al; St. Jude Children's Research Hospital-Washington University Pediatric Cancer Genome Project. Targeting oxidative stress in embryonal rhabdomyosarcoma. Cancer Cell 2013;24:710-24. DOI PubMed PMC

7. Horton JK, Houghton PJ, Houghton JA. Reciprocal cross-resistance in human rhabdomyosarcomas selected in vivo for primary resistance to vincristine and L-phenylalanine mustard. Cancer Res 1987;47:6288-93. PubMed

8. Houghton JA, Houghton PJ, Brodeur GM, Green AA. Development of resistance to vincristine in a childhood rhabdomyosarcoma growing in immune-deprived mice. Int J Cancer 1981;28:409-15. DOI PubMed

9. Jones M, Siracky J, Kelland LR, Harrap KR. Acquisition of platinum drug resistance and platinum cross resistance patterns in a panel of human ovarian carcinoma xenografts. Br J Cancer 1993;67:24-9. DOI PubMed PMC

10. Yamamoto TM, McMellen A, Watson ZL, et al. Activation of Wnt signaling promotes olaparib resistant ovarian cancer. Mol Carcinog 2019;58:1770-82. DOI PubMed PMC

11. Gay CM, Stewart CA, Park EM, et al. Patterns of transcription factor programs and immune pathway activation define four major subtypes of SCLC with distinct therapeutic vulnerabilities. Cancer Cell 2021;39:346-360.e7. DOI PubMed PMC

12. Ricci F, Brunelli L, Affatato R, et al. Overcoming platinum-acquired resistance in ovarian cancer patient-derived xenografts. Ther $A d v$ Med Oncol 2019;11:1758835919839543. DOI PubMed PMC

13. Kim K, Hu W, Audenet F, et al. Modeling biological and genetic diversity in upper tract urothelial carcinoma with patient derived xenografts. Nat Commun 2020;11:1975. DOI PubMed PMC

14. Ghilu S, Kurmasheva RT, Houghton PJ. Developing new agents for treatment of childhood cancer: challenges and opportunities for preclinical testing. J Clin Med 2021;10:1504. DOI PubMed PMC

15. Houghton PJ, Morton CL, Tucker C, et al. The pediatric preclinical testing program: description of models and early testing results. Pediatr Blood Cancer 2007;49:928-40. DOI PubMed

16. Houghton P, Kurmasheva R, Erickson S, et al. Prospective validation of single mouse testing (SMT) by the pediatric preclinical testing consortium (PPTC). Eur J Cancer 2020;138:S18. DOI

17. Ghilu S, Li Q, Fontaine SD, et al. Prospective use of the single-mouse experimental design for the evaluation of PLX038A. Cancer Chemother Pharmacol 2020;85:251-63. DOI PubMed PMC

18. Murphy B, Yin H, Maris JM, et al. Evaluation of alternative in vivo drug screening methodology: a single mouse analysis. Cancer Res 2016;76:5798-809. DOI PubMed PMC

19. Rokita JL, Rathi KS, Cardenas MF, et al. Genomic profiling of childhood tumor patient-derived xenograft models to enable rational clinical trial design. Cell Rep 2019;29:1675-89.e9. DOI PubMed PMC

20. Stewart E, Federico S, Karlstrom A, et al. The childhood solid tumor network: a new resource for the developmental biology and oncology research communities. Dev Biol 2016;411:287-93. DOI PubMed PMC

21. Peng KW, Myers R, Greenslade A, et al. Using clinically approved cyclophosphamide regimens to control the humoral immune response to oncolytic viruses. Gene Ther 2013;20:255-61. DOI PubMed PMC

22. Nair AB, Jacob S. A simple practice guide for dose conversion between animals and human. J Basic Clin Pharm 2016;7:27-31. DOI PubMed PMC

23. Crom WR, de Graaf SS, Synold T, et al. Pharmacokinetics of vincristine in children and adolescents with acute lymphocytic leukemia. J Pediatr 1994;125:642-9. DOI PubMed

24. Houghton PJ, Cheshire PJ, Myers L, Stewart CF, Synold TW, Houghton JA. Evaluation of 9-dimethylaminomethyl-10hydroxycamptothecin against xenografts derived from adult and childhood solid tumors. Cancer Chemother Pharmacol 1992;31:22939. DOI PubMed

25. Pappo AS, Lyden E, Breitfeld P, et al; Children's Oncology Group. Two consecutive phase II window trials of irinotecan alone or in combination with vincristine for the treatment of metastatic rhabdomyosarcoma: the Children's Oncology Group. J Clin Oncol 2007;25:362-9. DOI PubMed

26. Hawkins DS, Chi YY, Anderson JR, et al. Addition of vincristine and irinotecan to vincristine, dactinomycin, and cyclophosphamide does not improve outcome for intermediate-risk rhabdomyosarcoma: a report from the children's oncology group. J Clin Oncol 2018;36:2770-7. DOI PubMed PMC

27. Mascarenhas L, Meyer WH, Lyden E, et al; Soft Tissue Sarcoma Committee; Children's Oncology Group. Randomized phase II trial of bevacizumab and temsirolimus in combination with vinorelbine (V) and cyclophosphamide (C) for first relapse/disease progression of rhabdomyosarcoma (RMS): a report from the Children's Oncology Group (COG). J Clin Oncol 2014;32:10003. DOI

28. Minard-Colin V, Ichante JL, Nguyen L, et al. Phase II study of vinorelbine and continuous low doses cyclophosphamide in children and young adults with a relapsed or refractory malignant solid tumour: good tolerance profile and efficacy in rhabdomyosarcoma--a report from the Société Française des Cancers et leucémies de l'Enfant et de l'adolescent (SFCE). Eur J Cancer 2012;48:2409-16. DOI PubMed

29. Kuttesch JF, Parham DM, Luo X, et al. P-glycoprotein expression at diagnosis may not be a primary mechanism of therapeutic failure in childhood rhabdomyosarcoma. J Clin Oncol 1996;14:886-900. DOI PubMed

30. Hettmer S, Archer NM, Somers GR, et al. Anaplastic rhabdomyosarcoma in TP53 germline mutation carriers. Cancer 2014;120:106875. DOI PubMed PMC

31. Qualman S, Lynch J, Bridge J, et al. Prevalence and clinical impact of anaplasia in childhood rhabdomyosarcoma : a report from the Soft Tissue Sarcoma Committee of the Children's Oncology Group. Cancer 2008;113:3242-7. DOI PubMed PMC 
32. Rosenberg AR, Anderson JR, Lyden E, et al. Early response as assessed by anatomic imaging does not predict failure-free survival among patients with Group III rhabdomyosarcoma: a report from the Children's Oncology Group. Eur J Cancer 2014;50:816-23. DOI

33. Vaarwerk B, van der Lee JH, Breunis WB, et al. Prognostic relevance of early radiologic response to induction chemotherapy in pediatric rhabdomyosarcoma: a report from the International Society of Pediatric Oncology Malignant Mesenchymal Tumor 95 study. Cancer 2018;124:1016-24. DOI PubMed

34. Wang L, Hensch NR, Bondra K, et al. SNAI2-mediated repression of BIM protects rhabdomyosarcoma from ionizing radiation. Cancer Res 2021;81:5451-63. DOI PubMed PMC 